\title{
A computational study of the interface interaction between SARS-CoV-2 RBD and ACE2 from human, cat, dog and ferret.
}

\author{
William Sote ${ }^{1}$, Eduardo Franca ${ }^{1}$, Aline Hora ${ }^{2}$, and Moacyr Comar ${ }^{1}$ \\ ${ }^{1}$ Universidade Federal de Uberlandia \\ ${ }^{2}$ Federal University of Uberlandia
}

March 10, 2021

\begin{abstract}
The total impact of the worldwide COVID-19 pandemic is still emerging, changing all relationships as a result, including those with pet animals. In the infection process, the use of Angiotensin-converting enzyme 2 (ACE2) as a cellular receptor to the spike protein of the new coronavirus is a fundamental step. In this sense, understanding which residue plays what role in the interaction between SARS-CoV-2 spike glycoprotein and ACE2 from cats, dogs, and ferrets is an important guide for helping to choose which animal model can be used to study the pathology of COVID-19 and if there are differences between these interactions and those occurring in the human system. Hence, trying to help to answer these questions, we performed classical molecular dynamics simulations to evaluate, from an atomistic point of view, the interactions in these systems. Our results show that there are significant differences in the interacting residues between the systems from different animal species, and the role of ACE2 key residues are different in each system and can assist in the search for different inhibitors for each animal.
\end{abstract}

A computational study of the interface interaction between SARS-CoV-2 RBD and ACE2 from human, cat, dog and ferret.

William O. Soté ${ }^{1}$, Eduardo F. Franca ${ }^{1}$, Aline S. Hora ${ }^{2}$, Moacyr C. Junior ${ }^{1,{ }^{*}}$

${ }^{1}$ Chemistry Institute, Federal University of Uberlandia, Minas Gerais, Brazil

${ }^{2}$ School of Veterinary Medicine, Federal University of Uberlandia, Minas Gerais, Brazil

* Corresponding author: mcomjr@gmail.com

\begin{abstract}
The total impact of the worldwide COVID-19 pandemic is still emerging, changing all relationships as a result, including those with pet animals. In the infection process, the use of Angiotensin-converting enzyme 2 (ACE2) as a cellular receptor to the spike protein of the new coronavirus is a fundamental step. In this sense, understanding which residue plays what role in the interaction between SARS-CoV-2 spike glycoprotein and ACE2 from cats, dogs, and ferrets is an important guide for helping to choose which animal model can be used to study the pathology of COVID-19 and if there are differences between these interactions and those occurring in the human system. Hence, trying to help to answer these questions, we performed classical molecular dynamics simulations to evaluate, from an atomistic point of view, the interactions in these systems. Our results show that there are significant differences in the interacting residues between the systems from different animal species, and the role of ACE2 key residues are different in each system and can assist in the search for different inhibitors for each animal.
\end{abstract}

Keywords : SARS-CoV-2; RBD; ACE2; Molecular Dynamics

\section{Introduction}


The emergence of a new coronavirus ${ }^{1-4}$ with high infection capacity clearly shows that there is an increasing need to study many aspects of this pathogen. A key point during infection is cellular attachment of the virus, mediated by interaction between the SARS-CoV-2 receptor binding domain (RBD), which plays a pivotal role in host selectivity ${ }^{5,6}$, and host angiotensin-converting enzyme 2 (ACE2) receptor. The origin of the new coronavirus has been demonstrated to be a bat species ${ }^{7,8}$, but recent reports suggested that animals which share some specific residues in ACE2 could be hosts of SARS-CoV2, including cats, dogs and ferrets ${ }^{9}$. Hence, considering the large population and economic importance of companion animals ${ }^{10}$, and the difference in the ACE2 sequences (Fig 1), it is important to understand what is the role of these ACE2 key residues in the interaction process between SARS-CoV-2 RBD and ACE2, because the participation of companion animals in the epidemiological chain of COVID-19 transmission remains in debate ${ }^{11-14}$.

\section{FIGURE 1}

In this sense, evaluate this process from an atomistic point of view can provide important information about the role of these specific residues and the use of in silico strategies to simulate the interaction of proteins is ubiquitous, mainly using molecular docking and molecular dynamics simulations, since the advance of both hardware and software has allowed the study of bigger and larger systems ${ }^{15}$. In the case of the pandemic of the new coronavirus, molecular docking and classical molecular simulation are used in the some works to evaluate different aspects of the virus ${ }^{16-18}$. Considering the specific differences in the ACE2, we performed homology modeling, molecular docking and molecular dynamics simulations to observe the behavior of the interaction interface involving the RBD and ACE2 proteins for human, cat, dog, and ferret systems and, this is, as far we know, the first study of this kind.

\section{Materials and Methods}

\section{Homology modeling and docking procedures}

The structure used for human system was the SARS-CoV-2 receptor binding domain complexed with human receptor ACE2 (PDB: 6LZG). For cat (Uniprot: Q56H28), for dog (Uniprot: J9P7Y2), and for ferret (Uniprot: Q2WG88) systems the FASTA sequences of ACE2 receptors were used in the Swiss Model webserver ${ }^{19}$ to build receptor models that were equilibrated by a $1 \mathrm{~ns}$ simulation. The quality of all models was confirmed by Ramachandran plots (Supplementary material, Figure S1), using PROCHECK (LASKOWSKI et al., 1993). The amino acid sequence of the SARS-CoV-2 spike obtained from human (Uniprot: P0DTC2) was compared with those obtained from dogs (GenBank: QIT08256.1; QIT08292.1) and cats (GenBank; QOF07648.1; QLG96797.1) naturally infected with SARS-CoV-2 to assess possible differences in RDB by visual comparison after alignment using the BioEdit 7.0.5.3 software. ${ }^{20}$ No SARS-CoV-2 spike sequences from naturally infected ferrets were found in public database. In RDB, differences between amino acids from human, dog and cat were not found (Supplementary material, Figure S2). Finally, the SARS-CoV-2 RBD and the non-human receptors were docked using the HADDOCK $2.4 .{ }^{21}$. The active residues of both proteins and the docking results are in the Table 1.

\section{TABLE 1}

\section{Molecular Dynamics simulations}

The systems were solvated using the TIP3P water model ${ }^{22}$ and neutralized with appropriate counter-ions. Simulations were carried out using GROMACS $2016.4{ }^{23}$ and OPLS-AA force field ${ }^{24}$. All systems were simulated in triplicate. The energy minimization consisted of 50,000 steps using a convergence criterion of maximum force of $1,000 \mathrm{~kJ} \mathrm{~mol}^{-1} \mathrm{~nm}^{-1}$ followed by two steps of equilibration with all non-hydrogen protein atoms constrained by a force constant of $1,000 \mathrm{~kJ} \mathrm{~mol}^{-1} \mathrm{~nm}^{-2}$. The first equilibration step consisted of simulation for $125 \mathrm{ps}$ of an isochoric-isothermal (NVT) ensemble at $310 \mathrm{~K}$ maintained by velocity-rescale coupling method ${ }^{25}$ with a coupling time constant of $0.1 \mathrm{ps}$; the second was used an isobaric-isothermal (NPT) ensemble at 1.0 bar isotropically applied and maintained by the Berendsen weak coupling method ${ }^{26}$ with a coupling time constant of $0.5 \mathrm{ps}$ and compressibility of $4.510^{-5} \mathrm{bar}^{-1}$ by the same amount of time. Each production step of $50 \mathrm{~ns}$ used a time step of $1 \mathrm{fs}$ with no position restraints; the temperature was maintained 
at $310 \mathrm{~K}$ by the Nosé-Hoover thermostat ${ }^{27,28}$ and pressure was maintained at 1.0 bar using a ParrinelloRahman barostat ${ }^{29,30}$. Long-range electrostatics were evaluated by Particle-Mesh Ewald (PME), and a 1.0 $\mathrm{nm}$ cut off was considered for short-range interactions. All analyses were performed with GROMACS and VMD $^{31}$.

\section{Results and Discussion}

In this work, homology modeling, molecular docking and molecular dynamics simulations involving SARSCoV-2 RBD and ACE2 from human, cat, dog, and ferret systems were used in order to evaluate the interaction interface in each system. All simulations were done in triplicate of $50 \mathrm{~ns}$ each, a total of $600 \mathrm{~ns}$ of simulation were done and the first analysis, showed in Figure 2, is the average Root-mean-square Fluctuation (RMSF) for ACE2 interacting residues (The results for all replicas are in the Figures S3 to S6). The behavior is almost the same for all structures, except for two regions, around the residues number 60 for canine system and 110 for feline system where the fluctuations are expressive. Considering the key residues of ACE2, K31, $\mathrm{E} 35, \mathrm{D} 38, \mathrm{M} 82$ and $\mathrm{K} 353^{32,33}$, the average fluctuation for human system residues is ten times lower than for the other systems, Table 2.

\section{FIGURE 2}

\section{TABLE 2}

This behavior and the average fluctuation of the residues for human system, suggest that human interface of interaction is more stable, which can also be confirmed by the formation and maintenance of hydrogen bonds along all simulation time, Figure 3 .

\section{FIGURE 3}

For cat and dog interaction interfaces, the average number of hydrogen bonds decrease as the simulation is performed, suggesting an adjustment of the residue's positions. For feline system, Figure S7, considering the three simulations, the behavior is almost the same, only varying the number of hydrogen bonding along the simulation time. For canine system, there are some differences between the simulations, mainly in the range of 7-15 ns, but the average behavior is the decreasing of the hydrogen bonding number, Figure S8. For ferret system, Figure S9, the differences between the simulations are more intense than that observed for feline and canine systems, because in one replica there are two moments, near $20 \mathrm{~ns}$ and $30 \mathrm{~ns}$, that there are no identifiable hydrogen bonds. These results suggest that, for ferret system, the adjustment of the interaction interface is greater than for the other systems. For human system, Figure S10, the number of hydrogen bonds along all simulations is basically constant and is similar with that found in a recent report ${ }^{34}$, suggesting that the interaction interface does not fluctuate as the other pet animals systems studied herein.

In these systems, key residues are considered crucial regions of interaction in the process of SARS-CoV and SARS-CoV-2 host cells infection ${ }^{32,33}$. Human ACE2 presents five key residues (K31, E35, D38, M82 and K353) while cats, dogs and ferrets present three (K31, E35 and K353), and an important question is to know what interactions these residues can make, indicating possible sites of inhibition. In order to identify the interactions of these residues we used the structures obtained from clustering of each replica. The interaction of all residues for the systems are presented in the Table S1 and the principal interactions are in the Figures S11-S14.

Considering the human system, in all replicas the key residues of ACE2 make interactions with RBD residues in agreement with results described by Wan et al. ${ }^{35}$ and Lan et al. ${ }^{36}$. Furthermore, two key residues, E35 and M82, each one interacts with only one RBD residue, Q493 and F486, respectively. All other residues make more than one interaction, but the RBD residues are basically the same in all replica. The only difference is for K31 that makes one interaction in replica 1, three and four in the others. A long discussion of the interaction of human ACE2, SARS-CoV and SARS-CoV-2 is in the work of Vijayan ${ }^{34}$.

Considering the dog system, the interactions presented the same behavior that was noted in other systems: residues from RBD and ACE2 formed more than one interaction. Two of three key residues, one is a hot 
spot, K31, were found making interactions in all replicas. The interaction pattern shows some residues from RBD that form more than one interaction, and could be considered as targets, such as R466, K462 and L517 for example. From ACE2, E328 and K67 could be considered targets as well. In the cat system among all interacting residues in the RBD, there are some that could be considered targets for inhibitors due to the number of interactions that they can form, such as E57, N330 and F72. However, as can be observed in Table $\mathrm{S} 1$, the number of interaction residues change between replicas, suggesting that the interaction interface is moving and changing the interacting residues. Considering the key residues, there are three of them, K31, E35 and K353. However, K31 only interact in replica number 3, which is different of the dog system, where K31 makes interaction in all three simulations. The residue K458 from RBD interacts with E35 and K353, that can be considered a target. Considering the ferret system, two key residues were found: K31 (a hot spot) and E35. The residue F486 from ferret's RBD seems to be a key residue because it forms several interactions, with different residues in all replicas. The interactions of the two key residues, K31V445 and K31G498, as well as E35G446, E35G447, and E35N448, suggest that the glycine residue can be a target for inhibitor development for this system, in addition to glutamine and phenylalanine residues.

Along with the analysis of interacting residues we extracted from simulations, ten most persistent hydrogen bonding and among them we show for how long the key residues, if they appear, maintained its interactions, Figure 4 .

\section{FIGURE 4}

For cat system, the hydrogen bonds occupancy shows that only E35 key residue is found in them and presents a maximum occupancy of $71.73 \%$ as acceptor of hydrogen bonding in one replica and the donor was the K458 from RBD, that was the most common interacting residue to E35 along with G476 as well, although the occupancy with this residue is lower than $50 \%$ of the simulation time. Other interactions for cat system involving E35 residue are with K458 and Q474; the K353 residue make interactions with N460 and K458 and K31 do not make any interactions. From dog system, E35 was the most common interacting residue, as observed in the cat system. However, the occupancy was far below that observed for cat interface, maintaining in all simulations below $50 \%$. This residue interacts in a groove formed by S470 and T471 residues and T471 interact with other ACE2 key residue, E31 that showed and occupancy of almost $40 \%$ in the first replica, but not appeared in other two. The K31 For ferret system, the only ACE2 key residue to figure among the first ten with higher occupancy, is E35, showing a maximum value of $90.51 \%$, when interacting with G446, and make interactions with N448 (79.20\%), G447 (67.86\%) as well. Another characteristic for ferret system is the remarkable difference between replicas, suggesting that this interface is not well stablished. For human system however, almost all ACE2 key residues are present, showing occupancy above 33\%. Residues D38 and K353 interact with the same RDB residue, Q498, but only D38 interacts with Y499. The key residues E35, D38 and K353 present the more stable interactions, forming a group that can contribute to the stable interface interaction. The only residue that was not found doing any interaction is T82.

The COVID-19 pandemic caused by SARS-CoV-2 infections has created an urgent need for treatment and vaccine research, which require testing in appropriate animal models of the disease. An ideal model is one in which the pattern of infection is similar to that which occurs in humans ${ }^{37}$. Our results suggest that the use of dog, cat, or ferret as experimental models will not reflect what happens during infection in humans, so results obtained with in vivo experiments with these species may not be applicable to addressing COVID-19. Additionally, Bao ${ }^{38}$ confirmed experimentally that the pathogenicity of SARS-CoV-2 was reproduced only in transgenic mice that express human ACE2 (hACE2), and not in wild-type mice, concluding that hACE2 was essential for SARS-CoV-2 infection and replication in mice. Dogs and cats do not suffer from the presence of SARS-CoV-2, showing an absence of the persistent infection and no clinical signs ${ }^{12,39}$, which is explained from an atomistic point of view by the differences in interactions observed in our results. Furthermore, SARS$\mathrm{CoV}-2$ leads to acute bronchiolitis in experimentally infected ferrets; however, fatalities were not observed ${ }^{40}$, and reports of natural infections in this species were not found; high-affinity virus receptor interaction might be one of the crucial factors that determines virulence of this pathogen in the host ${ }^{41}$.

Beyond the question of these species as animal models, the possibility of detection of SARS-CoV-2 in com- 
panion animals has generated concern about abandonment ${ }^{14,42}$. Cat and dog populations are extremely large in several countries, and the increase of these animals circulating in the streets could result in serious consequences to public health, in addition to questions related to animal welfare. Results obtained in this work suggest very low-level participation of these animals in pandemic maintenance, which is supported by reports that natural infections are rarely observed in dogs and cats (fewer than 25 cases) and absent in ferrets.

\section{Conclusions}

In this work, molecular dynamics simulations were used to study the interaction interface between SARSCoV-2-RBD and ACE2 from humans, cats, dogs, and ferrets in order to identify commonalities across species. The analysis of the structural results suggest that the interaction interface of human system is more stable, corroborated by differences of the fluctuation values for the key residues and the behavior of the hydrogen bonds along the simulation time. The differences in the number of interactions, made by key residues during the replicas, between the systems suggest that the infection process in humans is more effective. Additionally, the hydrogen bonds formed time presents high differences in the occupancy time during the simulation, showing that the human RDB-ACE2 interface can be considered more stable among the studied systems. Furthermore, the few reports of natural occurrence of SARS-CoV-2 in pet animals worldwide suggest that infection in these animals rarely occurs, and the results discussed here can help evaluate the reasons for these numbers. In addition, our data suggest that the use of dogs, cats, and ferrets as experimental models for treatment and vaccines for SARS-CoV-2 should be considered cautiously, if not actively discouraged. Potential inhibitors of the SARS-CoV-2/ACE2 interaction must be considered to target additional residues along with the canonical key residues of RBD, as we show for human, cat, dog, and ferret.

\section{Conflict of interest}

The authors confirm that there is no conflict of interest.

\section{References}

1. Zhou, P.; Yang, X. L. X.-L.; Wang, X. G. X.-G.; Hu, B.; Zhang, L.; Zhang, W.; Si, H. R. H.-R.; Zhu, Y.; Li, B.; Huang, C.-L. C. L.; Chen, H. D. H.-D.; Chen, J.; Luo, Y.; Guo, H.; Jiang, R. D. R.-D.; Liu, M. Q. M.-Q.; Chen, Y.; Shen, X. R. X.-R.; Wang, X. G. X.-G.; Zheng, X.-S. X. S.; Zhao, K.; Chen, Q. J. Q.-J.; Deng, F.; Liu, L.-L. L. L.; Yan, B.; Zhan, F.-X. F. X.; Wang, Y.-Y. Y. Y.; Xiao, G. F. G.-F.; Shi, Z. L. Z.-L. ;Nature 2020 , $579,270$.

2. Wu, F.; Zhao, S.; Yu, B.; Chen, Y.-M.; Wang, W.; Song, Z.-G.; Hu, Y.; Tao, Z.-W.; Tian, J.-H.; Pei, Y.-Y.; Yuan, M.-L.; Zhang, Y.-L.; Dai, F.-H.; Liu, Y.; Wang, Q.-M.; Zheng, J.-J.; Xu, L.; Holmes, E. C.; Zhang, Y.-Z. ; Nature 2020, 579, 265.

3. Zhu, N.; Zhang, D.; Wang, W.; Li, X.; Yang, B.; Song, J.; Zhao, X.; Huang, B.; Shi, W.; Lu, R.; Niu, P.; Zhan, F.; Ma, X.; Wang, D.; Xu, W.; Wu, G.; Gao, G. F.; Tan, W.; Team, C. N. C. I. and R. ;N. Engl. J. Med. 2020 , 382, 727.

4. Morens, D. M.; Daszak, P.; Taubenberger, J. K. Escaping Pandora's box - Another novel coronavirus. N. Engl. J. Med. 2020 ,382, 1293-1295.

5. Li, W.; Moore, M. J.; Vasilieva, N.; Sui, J.; Wong, S. K.; Berne, M. A.; Somasundaran, M.; Sullivan, J. L.; Luzuriaga, K.; Greenough, T. C.; Choe, H.; Farzan, M. ;Nature 2003 , 426 , 450.

6. Li, F. ;J. Virol. 2015, 89,1954 LP.

7. Cui, J.; Li, F.; Shi, Z.-L. ;Nat. Rev. Microbiol.2019 , 17 , 181.

8. Li, W.; Wong, S.-K.; Li, F.; Kuhn, J. H.; Huang, I.-C.; Choe, H.; Farzan, M. ;J. Virol. 2006 , 80 , 4211 LP.

9. Luan, J.; Lu, Y.; Jin, X.; Zhang, L. ;Biochem. Biophys. Res. Commun. 2020 , 526 , 165. 
10. Gapper, J. Pets are making Wall Street wag its tail.

11. Shi, J.; Wen, Z.; Zhong, G.; Yang, H.; Wang, C.; Huang, B.; Liu, R.; He, X.; Shuai, L.; Sun, Z.; Zhao, Y.; Liu, P.; Liang, L.; Cui, P.; Wang, J.; Zhang, X.; Guan, Y.; Tan, W.; Wu, G.; Chen, H.; Bu, Z. ;Science $\mathbf{2 0 2 0}, 368,1016$.

12. Sit, T. H. C.; Brackman, C. J.; Ip, S. M.; Tam, K. W. S.; Law, P. Y. T.; To, E. M. W.; Yu, V. Y. T.; Sims, L. D.; Tsang, D. N. C.; Chu, D. K. W.; Perera, R. A. P. M.; Poon, L. L. M.; Peiris, M. ;Nature2020, 1.

13. Mallapaty, S. S. ; Nature 2020 .

14. da Hora, A. S. ; Nature $2020,580,321$.

15. Perilla, J. R.; Schulten, K. ;Nat. Commun. 2017 ,8 , 15959.

16. Basu, A.; Sarkar, A.; Maulik, U. ;Sci. Rep. 2020 ,10 , 17699.

17. Arantes, P. R.; Saha, A.; Palermo, G. ;ACS Cent. Sci.2020 , 6 , 1654.

18. Razzaghi-Asl, N.; Ebadi, A.; Shahabipour, S.; Gholamin, D. ;J. Biomol. Struct. Dyn. 2020 , 1.

19. Waterhouse, A.; Bertoni, M.; Bienert, S.; Studer, G.; Tauriello, G.; Gumienny, R.; Heer, F. T.; de Beer, T. A. P.; Rempfer, C.; Bordoli, L.; Lepore, R.; Schwede, T. ;Nucleic Acids Res. 2018 ,46 , W296.

20. Hall, T. A. ;Nucleic Acids Symp. Ser. No. 4195.

21. Van Zundert, G. C. P.; Rodrigues, J. P. G. L. M.; Trellet, M.; Schmitz, C.; Kastritis, P. L.; Karaca, E.; Melquiond, A. S. J.; Van Dijk, M.; De Vries, S. J.; Bonvin, A. M. J. J. ;J. Mol. Biol.2016 , 428 , 720.

22. Jorgensen, W. L.; Chandrasekhar, J.; Madura, J. D.; Impey, R. W.; Klein, M. L. ;J. Chem. Phys. 1983 , 79,926 .

23. Abraham, M. J.; Murtola, T.; Schulz, R.; Páll, S.; Smith, J. C.; Hess, B.; Lindah, E. ;SoftwareX 2015 $, 1-2,19$.

24. Jorgensen, W. L.; Tirado-Rives, J. ;J. Am. Chem. Soc.1988 , 110 , 1657.

25. Bussi, G.; Donadio, D.; Parrinello, M. ;J. Chem. Phys.2007, 126 .

26. Berendsen, H. J. C.; Postma, J. P. M.; Van Gunsteren, W. F.; Dinola, A.; Haak, J. R. ;J. Chem. Phys. $1984,81,3684$.

27. Nosé, S. ;J. Chem. Phys. $1984,81,511$.

28. Hoover, W. G. ;Phys. Rev. A 1985 , 31 , 1695.

29. Nosé, S.; Klein, M. L. ;Mol. Phys. 1983, 50 , 1055.

30. Parrinello, M.; Rahman, A. ;J. Appl. Phys. 1981 ,52, 7182.

31. Humphrey, W.; Dalke, A.; Schulten, K. ;J. Mol. Graph.1996 , 14 , 33.

32. Li, F.; Li, W.; Farzan, M.; Harrison, S. C. ;Science (80-. ).2005 , 309 , 1864.

33. Wan, Y.; Shang, J.; Graham, R.; Baric, R. S.; Li, F. ;J. Virol. 2020 , 94.

34. Ali, A.; Vijayan, R. ;Sci. Rep. 2020 , 10 , 14214.

35. Wan, Y.; Shang, J.; Graham, R.; Baric, R. S.; Li, F. ;J. Virol. 2020 , 94 .

36. Lan, J.; Ge, J.; Yu, J.; Shan, S.; Zhou, H.; Fan, S.; Zhang, Q.; Shi, X.; Wang, Q.; Zhang, L.; Wang, X. Structure of the SARS-CoV-2 spike receptor-binding domain bound to the ACE2 receptor. 
37. Cleary, S. J.; Pitchford, S. C.; Amison, R. T.; Carrington, R.; Robaina Cabrera, C. L.; Magnen, M.; Looney, M. R.; Gray, E.; Page, C. P. ;Br. J. Pharmacol. 2020.

38. Bao, L.; Deng, W.; Huang, B.; Gao, H.; Liu, J.; Ren, L.; Wei, Q.; Yu, P.; Xu, Y.; Qi, F.; Qu, Y.; Li, F.; Lv, Q.; Wang, W.; Xue, J.; Gong, S.; Liu, M.; Wang, G.; Wang, S.; Song, Z.; Zhao, L.; Liu, P.; Zhao, L.; Ye, F.; Wang, H.; Zhou, W.; Zhu, N.; Zhen, W.; Yu, H.; Zhang, X.; Guo, L.; Chen, L.; Wang, C.; Wang, Y.; Wang, X.; Xiao, Y.; Sun, Q.; Liu, H.; Zhu, F.; Ma, C.; Yan, L.; Yang, M.; Han, J.; Xu, W.; Tan, W.; Peng, X.; Jin, Q.; Wu, G.; Qin, C. ;Nature 2020 .

39. Margaret J. Hosie, Katrin Hartmann, Regina Hofmann-Lehmann, Diane D. Addie, Uwe Truyen, Herman Egberink, Séverine Tasker, Tadeusz Frymus, Maria Grazia Pennisi, K. M. SARS-Coronavirus (CoV)-2 and cats.

40. Kim, Y. Il; Kim, S. G.; Kim, S. M.; Kim, E. H.; Park, S. J.; Yu, K. M.; Chang, J. H.; Kim, E. J.; Lee, S.; Casel, M. A. B.; Um, J.; Song, M. S.; Jeong, H. W.; Lai, V. D.; Kim, Y.; Chin, B. S.; Park, J. S.; Chung, K. H.; Foo, S. S.; Poo, H.; Mo, I. P.; Lee, O. J.; Webby, R. J.; Jung, J. U.; Choi, Y. K. ;Cell Host Microbe 2020, 27, 704 .

41. Sarkar, J.; Guha, R. ; Vet. Res. Commun. 2020 , 1.

42. Huang, Q.; Zhan, X.; Zeng, X.-T. ;J. Travel Med.2020 , 27.

Table 1 - Haddock results for non-human systems. Z-score negative values indicate better docking of the structures.

\begin{tabular}{lll}
\hline System & HADDOCK score & z-score \\
\hline Cat & $-58.5 \pm 5.0$ & -2.2 \\
Dog & $-34.5 \pm 8.5$ & -2.2 \\
Ferret & $-19.8 \pm 8.9$ & -2.2 \\
Human (redocking) & $-10.5 \pm 6.7$ & -1.9 \\
\hline
\end{tabular}

Table 2 . Average RMSF for ACE2 key residues. (In bold are those that shared by all species)

\begin{tabular}{llllll}
\hline & Key residues & Key residues & Key residues & Key residues & Key \\
\hline & Average RMSF (nm) & Average RMSF (nm) & Average RMSF (nm) & Average RMSF (nm) & Ave \\
Species & K31 & $\mathbf{E 3 5}$ & D38 & M82 & 0.1216 \\
Human & 0.0833 & 0.0774 & 0.0787 & 0.1832 & 0.12 \\
Ferret & 0.1171 & 0.1246 & 0.1091 & 0.2078 & 0.14 \\
Cat & 0.1584 & 0.1361 & 0.1253 & 0.1623 & 0.16 \\
Dog & 0.1036 & 0.1006 & 0.1119 & & \\
\hline
\end{tabular}




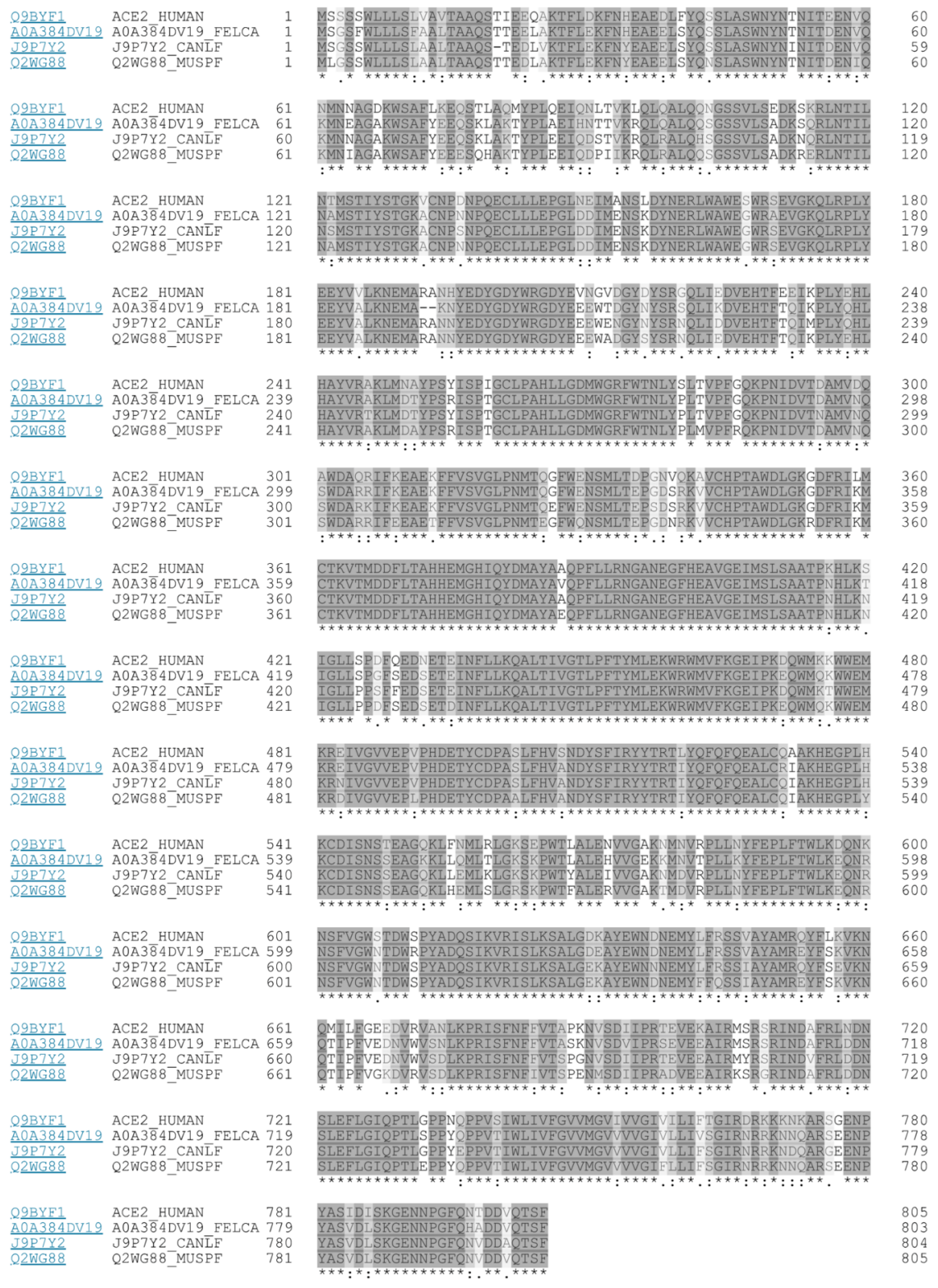

Figure 1 - Multiple sequence alignment of human (Q9BYF1), cat (A0A384DV19), dog (J9P7Y2) and ferret (Q2WG88) angiotensin-converting enzyme 2 (ACE2) receptor. Differences between sequences are light gray or white, considering sequence of human ACE2 as a reference. 


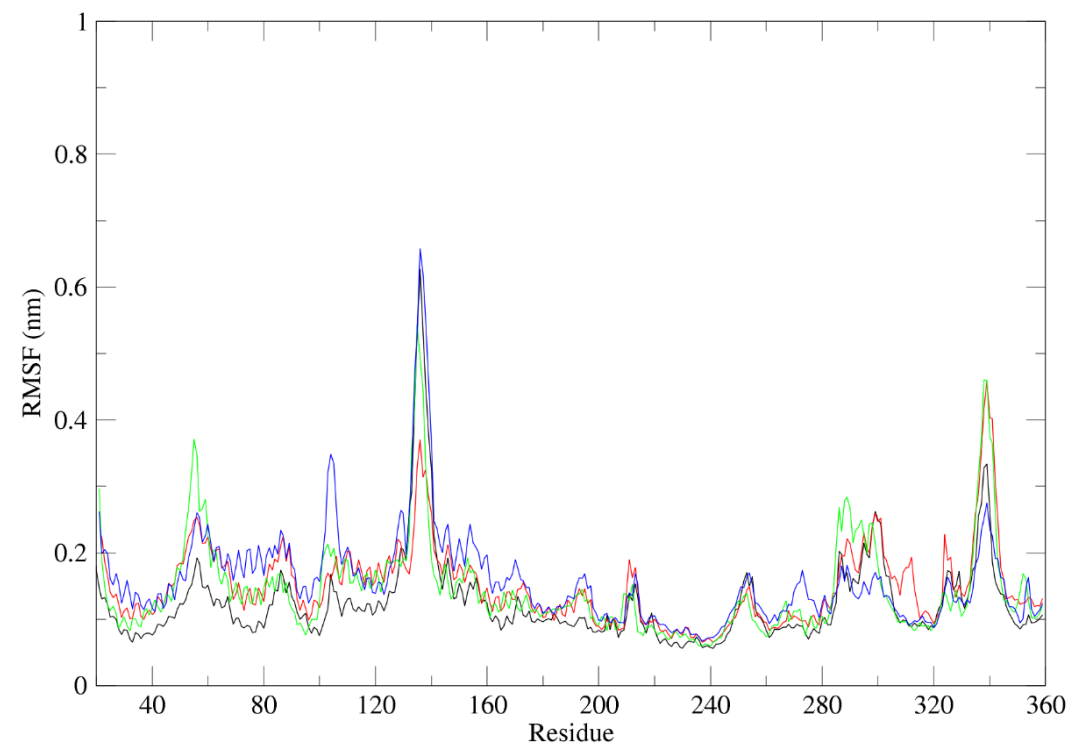

Figure 2 - Average RMSF for human (black), cat (blue), dog (green) and ferret (red). The complete graphs for replicas are in Supplementary Material (S3-S6).

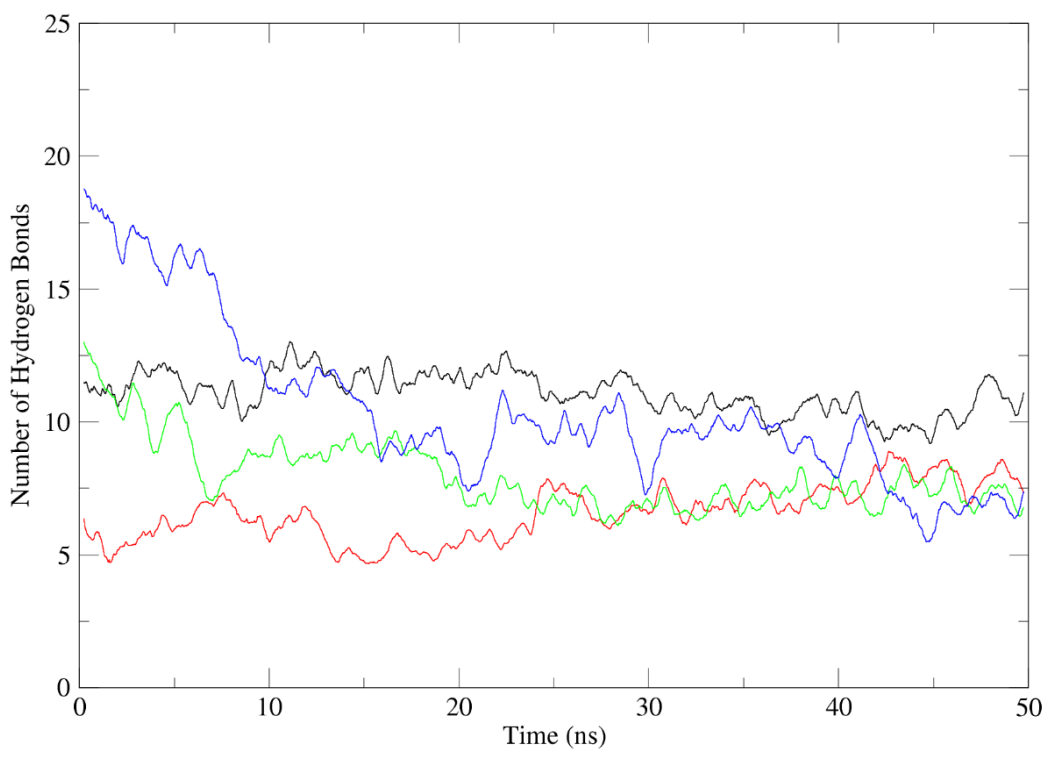


Figure 3 - Average number of hydrogen bond over time for the simulated systems. Human, cat, dog and ferret are shown in black, blue, green and red, respectively.
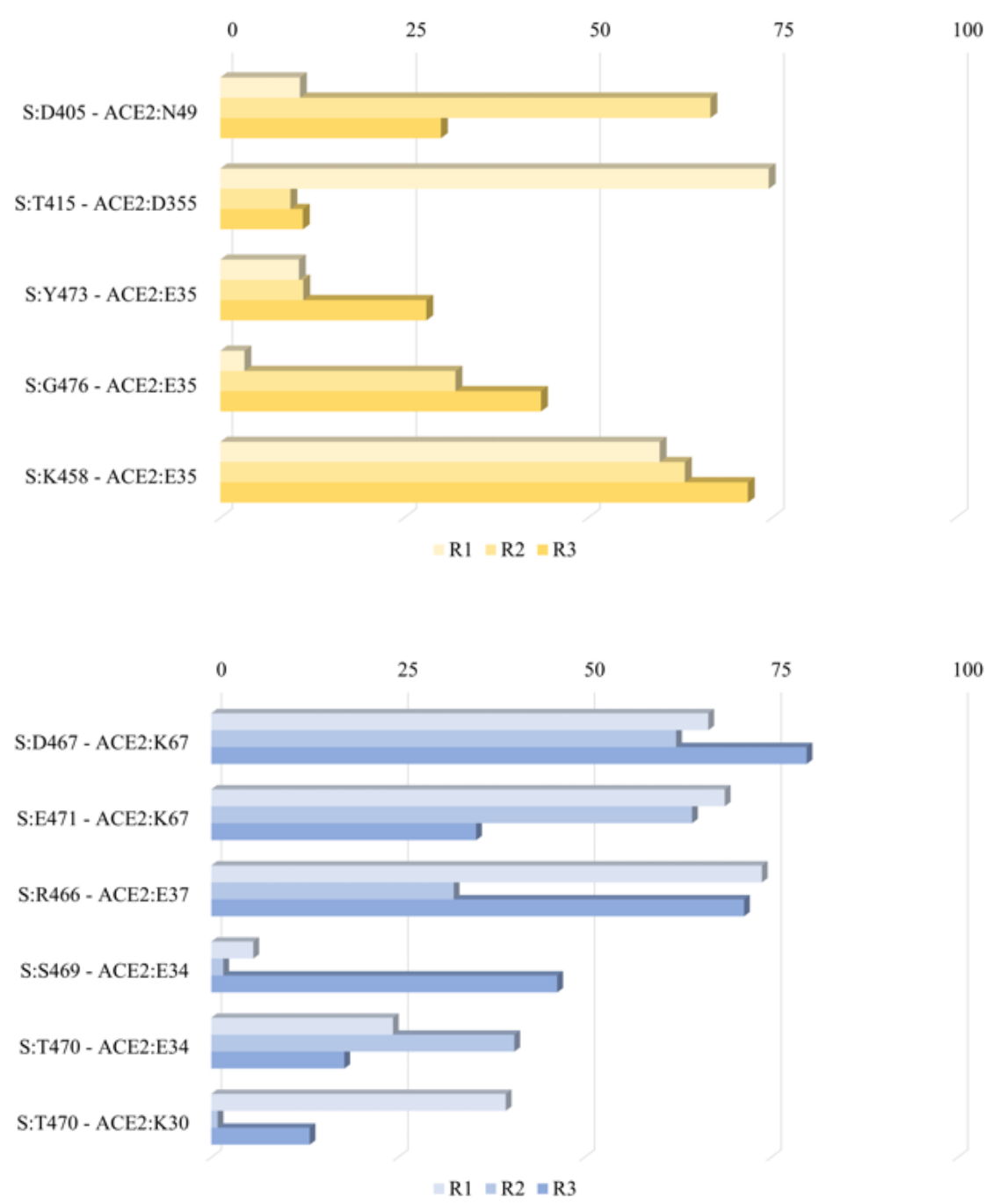

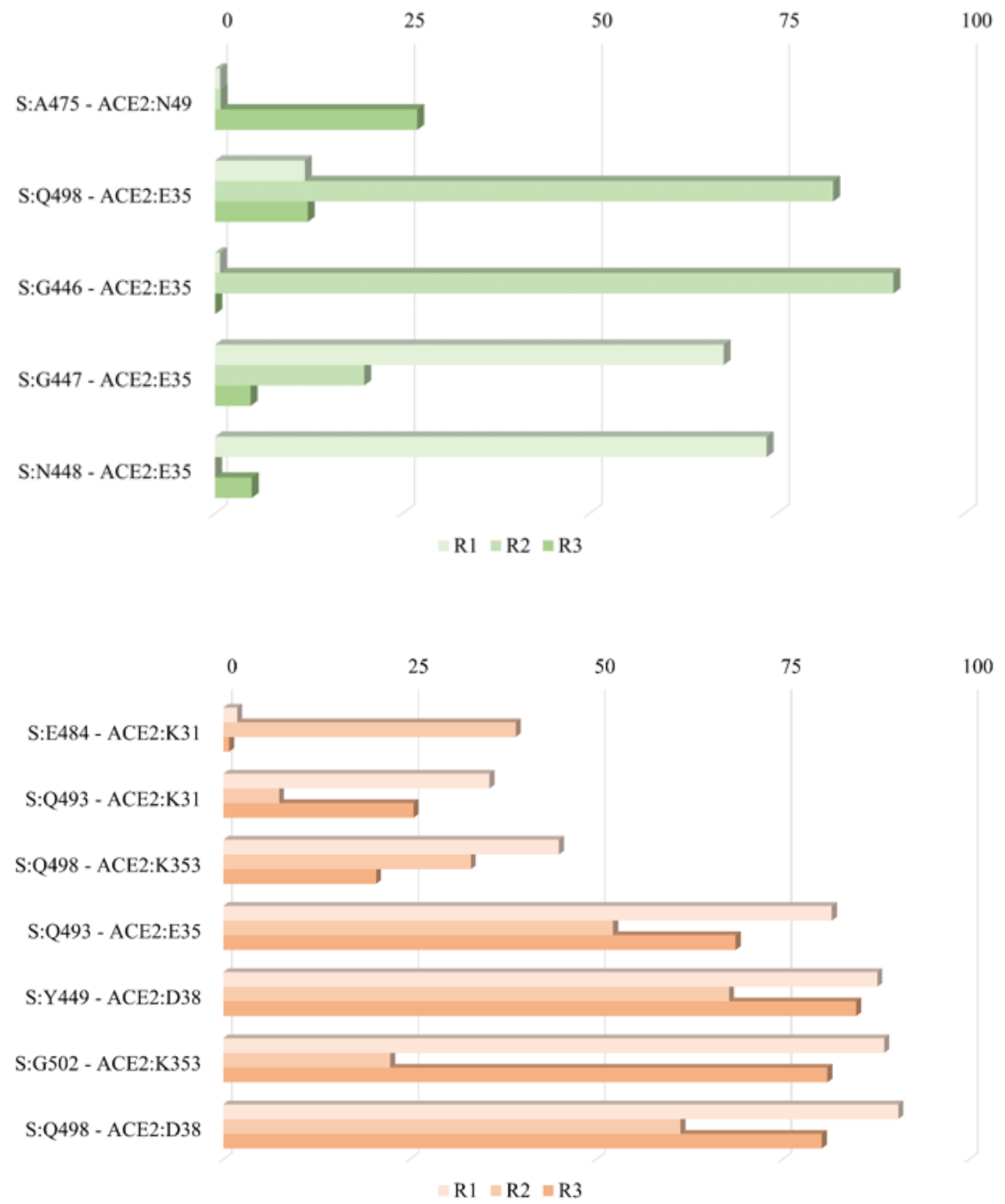

Figure 4 - Hydrogen bonds occupation during the simulation time through the replicas. (Yellow for cat system, blue for dog system, green for ferret system and orange for human system) 